\title{
ON THE MINIMAL PROPERTY OF THE FOURIER PROJECTION
}

\author{
BY E. W. CHENEY, ${ }^{1}$ C. R. HOBBY, ${ }^{1}$ P. D. MORRIS, ${ }^{2}$ \\ F. SCHURER ${ }^{3}$ AND D. E. WULBERT ${ }^{2}$
}

Communicated by Henry Helson, September 10, 1968

Let $C$ be the space of real $2 \pi$-periodic continuous functions normed with the supremum norm. Let $P_{n}$ denote the subspace of trigonometric polynomials of degree $\leqq n$. It is known [1] that the Fourier projection $F$ of $C$ onto $P_{n}$ is minimal; i.e., if $A$ is a projection of $C$ onto $P_{n}$ then $\|F\| \leqq\|A\|$. We prove that $F$ is the only minimal projection of $C$ onto $P_{n}$. The proof is constructed by verifying the assertions listed below. Details will appear elsewhere.

ASSERTION. If there exists a minimal projection different from $F$, then there exist minimal projections $L$ and $H$, different from $F$ such that $\frac{1}{2} L+\frac{1}{2} H=F$.

The proof of this assertion utilizes Berman's equation,

$$
F=\frac{1}{2 \pi} \int_{-\pi}^{\pi} T_{-\lambda} A T_{\lambda} d \lambda,
$$

which is valid for any projection $A$ of $C$ onto $P_{n}$. Here $T_{\lambda}$ denotes the shift operator $\left(T_{\lambda} f\right)(x)=f(x+\lambda)$.

ASSERTION. There is a function $K(x, t)$ of two variables such that

(i) $K(x, \cdot) \in L^{1}$ for each fixed $x$,

(ii) $K(\cdot, t) \in P_{n}$ for each fixed $t$, and

(iii) $(L f)(x)=\int f(t) K(x, t) d t$.

This is proved by extending $A$ to its second adjoint, and applying the Radon-Nikodym theorem to the functionals $\phi(f)=\left(A^{* *} f\right)(x)$.

Let $D_{n}$ denote the Dirichlet kernel. The next assertion follows from an examination of the roots of $K$ where $K$ is considered as a function of $x$.

Assertion. There is a function $g \in L^{1}$ such that $0 \leqq g \leqq 2$, and $K(x, t)=g(t) D_{n}(x-t)$.

AsSERTION. (i) $(1-g) \perp P_{2 n}$ and (ii) $(1-g) *\left|D_{n}\right|=0$ where $*$ denotes convolution.

1 Supported by the Air Force Office of Scientific Research.

- Supported by the National Science Foundation.

- Supported by a NATO Science Fellowship, granted by the Netherlands Organization for the Advancement of Pure Research (Z.W.O.). 
Part (i) is immediate from the fact that $L$ is a projection. The minimality of $L$ is needed to prove part (ii).

Let $d(n, k)=\int\left|D_{n}(t)\right| e^{i k t} d t$.

AsseRTION. $d(n, k) \neq 0$ for $|k|>2 n$.

This result, when combined with the preceding assertion, will prove the theorem. The remainder of this paper pertains to proving that $d(n, k) \neq 0$.

Assertion.

$$
d(n, k)=\frac{1}{\pi} \sum_{j=k-n}^{k+n} \frac{1}{j} \frac{\beta^{j}-1}{\beta^{j}+1}
$$

where $\beta=e^{2 \pi i / 2 n+1}$.

Assertion. If $d(n, k)=0$ then

$$
\sum_{j=k-n}^{k+n} \frac{1}{j} \sum_{t=1}^{2 n}\left(-\beta^{j}\right)^{t}=0 .
$$

Thus if $d(n, k)=0$ we have a polynomial of degree $2 n$ with rational coefficients which has $\beta$ as a root. We next derive a relation which must be satisfied by the coefficients of such a polynomial. The final step is to show that in our case this relation is not even satisfied modulo a convenient prime. The existence of the convenient prime is a consequence of the following extension of the Sylvester-Schur theorem.

AsSERTION. If $n$ and $k$ are integers satisfying $6 \leqq k \leqq n / 2$, then at least two integers between $n-k+1$ and $n$ possess prime factors exceeding $k$.

\section{REFERENCES}

1. D. L. Berman, On the impossibility of constructing a linear polynomial operator furnishing an approximation of the order of best approximation, Dokl. Akad. Nauk. SSSR 120 (1958), 143-148.

2. M. Golomb, Lectures on theory of approximation, Argonne National Laboratory, Argonne, Illinois, 1962. 1962.

3. K. Hoffman, Banach spaces of analytic functions, Prentice-Hall, New York,

University of Texas, Austin, Texas 78712

University of Washington, Seattle, Washington 98105

Pennsylvania State University, University Park, Pennsylvania 16802

Technological University, Eindhoven, Netherlands

University of Washington, Seattle, Washington 98105 